\title{
Temporal and spatial coherence properties of free-electron-laser pulses in the extreme ultraviolet regime
}

\author{
S. Roling, B. Siemer, M. Wöstmann, and H. Zacharias \\ Physikalisches Institut, Westfälische Wilhelms-Universität, 48149 Münster, Germany \\ R. Mitzner \\ Physikalisches Institut, Westfälische Wilhelms-Universität, 48149 Münster, Germany, \\ and Helmholtz-Zentrum Berlin für Materialien und Energie, 14109 Berlin, Germany \\ A. Singer, K. Tiedtke, and I. A. Vartanyants \\ Deutsches Elektronen-Synchrotron, Notkestrasse 85, 22603 Hamburg, Germany
}

(Received 10 March 2011; published 22 August 2011)

\begin{abstract}
The average temporal (longitudinal) and spatial (transverse) coherence of free-electron-laser pulses in the extreme ultraviolet at FLASH is measured by interfering two time-delayed partial beams directly on a CCD camera. Wavelengths between $\lambda=32 \mathrm{~nm}$ and $\lambda=8 \mathrm{~nm}$ are examined. A decrease of the coherence time for the fundamental wavelengths from $\tau_{c}=(6 \pm 0.5) \mathrm{fs}$ at $32 \mathrm{~nm}$ to $\tau_{c}=(2.9 \pm 0.5) \mathrm{fs}$ at $8 \mathrm{~nm}$ is measured. At $\lambda=8 \mathrm{~nm}$ the fundamental wavelength and the third harmonic of $24 \mathrm{~nm}$ are compared to each other. For $8 \mathrm{~nm}$ radiation as third harmonic of $24 \mathrm{~nm}$ a coherence time of $\tau_{c}=$ $(2.5 \pm 0.5)$ fs is observed. The spatial coherence of 24 and $8 \mathrm{~nm}$ fundamental pulses are found to be very similar. The visibility decreases to $50 \%$ of the maximum visibility at about $3.2 \mathrm{~mm}$ overlap of the partial beams, which corresponds to $42 \%$ of the beam diameter at a distance of $90 \mathrm{~m}$ from the exit of the undulator. These results are analyzed in terms of the Gaussian Schell model resulting in six contributing modes to the total radiation. In addition, the correlation of the visibility between the fundamental radiation at $24 \mathrm{~nm}$ and its third harmonic at $\lambda=8 \mathrm{~nm}$ is investigated for identical shots.
\end{abstract}

DOI: 10.1103/PhysRevSTAB.14.080701

PACS numbers: 41.60.Cr, 41.60.Ap, 41.50.+h, 42.25.Hz

\section{INTRODUCTION}

Among the light sources available in the extreme ultraviolet (XUV) and soft $\mathrm{x}$-ray regime self-amplified spontaneous emission (SASE) generated by free-electron lasers (FEL) provides widely tunable ultrashort light pulses with high pulse energies [1]. Since 2005 FLASH at DESY in Hamburg is operating as a user facility, by now providing radiation down to $4.12 \mathrm{~nm}$. In 2009 the first hard x-ray FEL Linac Coherent Lights Source (LCLS) started its operation in Stanford [2]. The intense radiation generated by such sources enables innovative experiments in various fields of research such as single shot coherent diffraction imaging [3,4], cluster physics [5], and surface reaction dynamics [6]. For the first type of experiments, in particular, but not only, a precise knowledge of the longitudinal and transverse coherence of the light pulses is required. Further, an understanding of the formation of coherence during the SASE process is important for the optimization of electron bunch formation. Substantial contributions to the theoretical description of the FEL process have been published in

Published by the American Physical Society under the terms of the Creative Commons Attribution 3.0 License. Further distribution of this work must maintain attribution to the author(s) and the published article's title, journal citation, and DOI. recent years [7-11]. Still an experimental verification of the essential temporal and spatial beam properties for different operating parameters of the FEL in particular at different wavelengths is of great interest. Recently, measurements of the average pulse duration of the free electron laser in Hamburg (FLASH) yields values around $30 \mathrm{fs}$, both by means of two-photon double ionization of helium [12] and for single shots by electric field streaking driven by $\mathrm{THz}$ radiation from synchronized undulator pulses [13]. First measurements of the temporal coherence of FLASH pulses at $\lambda=24 \mathrm{~nm}$ using a linear autocorrelation revealed a coherence time of $\tau_{c}=6 \mathrm{fs}$ and a multiple pulse substructure [14]. Schlotter et al. supplemented these findings at longer and shorter wavelengths of $\lambda=33.2 \mathrm{~nm}$ and $\lambda=9.6 \mathrm{~nm}$ [15]. Measurements of the spatial coherence of FLASH pulses applying a Young double-slit experiment at $\lambda=13.7 \mathrm{~nm}$ at a distance of $z=20 \mathrm{~m}$ from the source showed a coherence length of about $\xi=(300 \pm 15) \mu \mathrm{m}$ in the horizontal and vertical directions. The beam radius (half width at half maximum of a Gaussian function) at this distance from the source is about $890 \mu \mathrm{m}$ [16].

In this paper a characterization of the temporal and spatial coherence of FLASH generating fs pulses at different wavelengths from $\lambda=32 \mathrm{~nm}$ to $\lambda=8 \mathrm{~nm}$ is presented in order to investigate the dependence of the coherence properties as a function of wavelength. The 
results are compared to theoretical predictions. In addition, the temporal coherence of third harmonic radiation at $\lambda=8 \mathrm{~nm}$ is determined when FLASH is set to operate at the fundamental at $\lambda=24 \mathrm{~nm}$.

\section{EXPERIMENTAL}

As an essential prerequisite for coherence measurements, one has to ensure that the overlapping partial beams do not show any jitter both temporally and spatially. In the present setup this is achieved by single pulse measurements at defined spatial overlap and time delay. To provide such jitter-free replica pulses for $\mathrm{x}$-ray pump/x-ray probe experiments, a split-and-delay unit (autocorrelator) has been developed for the use with FLASH, based on geometrical wave front beam splitting by a sharp mirror edge. Employing grazing incident angles for the XUV radiation it covers the fundamental photon energy range of FLASH from $h \nu=20 \mathrm{eV}$ to more than $200 \mathrm{eV}$ with an efficiency of larger than $50 \%$. A schematic drawing of the layout of the autocorrelator is shown in Fig. 1. Grazing angles of $3^{\circ}$ and $6^{\circ}$ for the fixed and variable delay arms, respectively, are employed to ensure a high reflectivity in the soft $\mathrm{x}$-ray regime. The left part of the incoming FEL pulse is reflected horizontally by the beam splitter with a sharp edge into the fixed beam path. The other part of the beam passes the beam splitter unaffected and is then reflected vertically by the second mirror into a variable delay line. There, a delay between -5 and +20 ps with respect to the fixed beam path can be achieved with a step size of nominally 40 as. The seventh and eighth mirrors reflect the partial beams again into their original direction. Small angles between

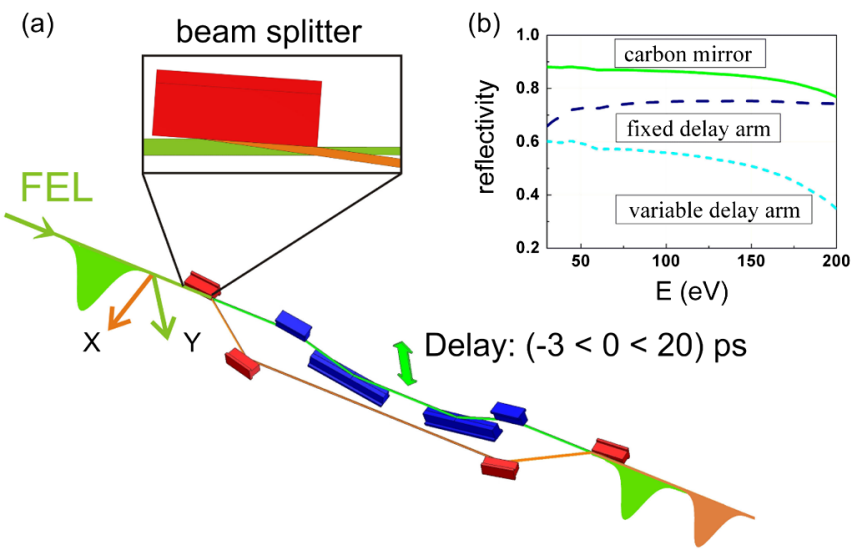

FIG. 1. Scheme of the autocorrelator. (a) The FEL pulse is divided by the wave front beam splitter. One partial beam is reflected horizontally into a fixed arm of the autocorrelator, the other partial beam passes the beam splitter and is vertically reflected into the delay stage. With the last mirror the beams are recombined. Different overlap angles on a CCD camera can be set by rotating the last mirror. (b) The reflectivity of a carbon coated silicon mirror is shown as a function of photon energy (green line). The dashed lines represent the total transmission of the fixed (dark blue) and the variable delay arm (light blue). both beams can be introduced in order to vary the spatial overlap. Consequently, the distance between two points of the spatial beam profile interfering with each other is varied. Thus, not only the temporal but also the spatial coherence of the FEL pulses can be measured. A more detailed description of the autocorrelator can be found in [14]. The autocorrelator is assembled at beam line BL3 at a distance of $70 \mathrm{~m}$ downstream from the undulator. The coherence properties of FEL pulses with wavelengths of $\lambda=32,24,13,8$ (fundamental), and $8 \mathrm{~nm}$ (third harmonic of $\lambda=24 \mathrm{~nm}$ ) are investigated.

The experimental setup is shown in Fig. 2. In order to increase the propagation distance behind the autocorrelator both partial beams are reflected by a plane multilayer mirror $(\mathrm{Mo} / \mathrm{Si})$ under an angle of incidence of $\theta=10^{\circ}$ directly onto a XUV sensitive CCD camera at a distance of $20 \mathrm{~m}$ behind the autocorrelator. The mirror shows a reflectivity of $R_{1}=3.0 \%$ at $\lambda=8 \mathrm{~nm}$ and $R_{2}=2.0 \%$ at $\lambda=24 \mathrm{~nm}$, with spectral bandwidths of $\Delta \lambda=0.5 \mathrm{~nm}$ and more than $1.5 \mathrm{~nm}$ at $\lambda=8$ and $24 \mathrm{~nm}$, respectively. For $\lambda=13 \mathrm{~nm}$ and $\lambda=32 \mathrm{~nm}$ a mirror coated with chromium was used, showing a reflectivity of $R_{3}=0.14 \%$ at $\lambda=13 \mathrm{~nm}$ and $R_{4}=0.9 \%$ at $\lambda=32 \mathrm{~nm}$, respectively. The low reflectivity of the mirrors was used in order to attenuate the FEL radiation so that the CCD camera is not destroyed. Further attenuation is achieved by a gas absorber filled with $\mathrm{N}_{2}$ to ensure that the full dynamic range of the 16 bit CCD is available. Because of the finite readout time of the $\mathrm{CCD}$, a fast shutter is used to separate individual FEL pulses. The initial repetition rate of $5 \mathrm{~Hz}$ is thereby reduced to $0.5 \mathrm{~Hz}$.

The partial beams are brought to an overlap directly on the chip of the CCD camera at a distance of $L_{d}=20 \mathrm{~m}$ from the last mirror of the autocorrelator. This distance is required to ensure that a sufficient number of pixels of the CCD camera are illuminated by one interference fringe.

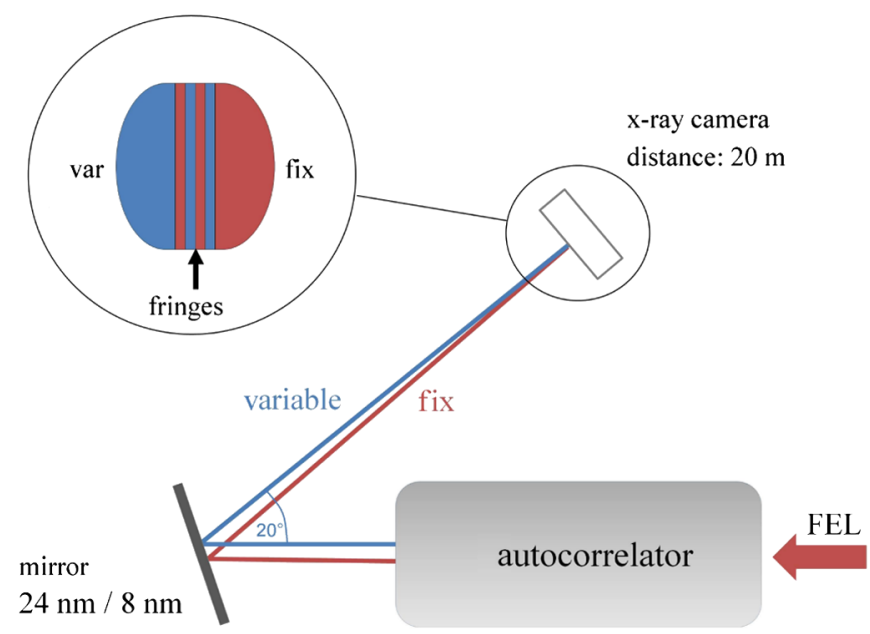

FIG. 2. The experimental setup: Behind the autocorrelator the partial beams are reflected back by a multilayer mirror to overlap at a distance of $20 \mathrm{~m}$ on an $\mathrm{x}$-ray CCD camera. 
The width $d$ of the interference fringes observed is inversely proportional to the angle $\alpha$ under which the partial beams overlap, according to

$$
d=\frac{\lambda}{\sin \alpha} \text {. }
$$

For a wavelength of $\lambda=8 \mathrm{~nm}$ and a typical angle of $\alpha=70 \mu \mathrm{rad}$, this yields a spacing of the interference fringes of $d=114 \mu \mathrm{m}$. The spatial resolution of the CCD camera amounts to $s=13.5 \mu \mathrm{m}$ per pixel. Thus, even for short wavelengths each interference fringe is sampled by about eight pixels. For the measurements of the spatial coherence, the overlap angle is increased up to $200 \mu \mathrm{rad}$ yielding a fringe width of only $40 \mu \mathrm{m}$ with about three pixels per fringe. A decrease of the ratio between the width of the interference fringes $d$ and the pixel size $s$ (sampling rate) causes a degradation of the measured visibility compared to the real visibility by a factor $k$, which is defined by the modulation transfer function (MTF) of the camera due to the pixel size. This factor has been calculated and used to correct the visibility, see Fig. 3.

For the measurement of the harmonic radiation a suppression of the intensity of the fundamental radiation is necessary. Therefore, a $\mathrm{Zr}$ filter $(d=200 \mathrm{~nm})$ with a transmission of $T=55 \%$ at $\lambda=8 \mathrm{~nm}$ and $T=0.02 \%$ at $\lambda=$ $24 \mathrm{~nm}$ was used. In order to detect interference fringes from the fundamental and harmonic radiation simultaneously D-shaped filters are applied, which block only part of the beam. Here again $\mathrm{Zr}$ is used to suppress the fundamental, while $\mathrm{Al}(d=400 \mathrm{~nm}, T=49 \%$ for $\lambda=$ $24 \mathrm{~nm}$ and $T=3 \times 10^{-3} \%$ for $\lambda=8 \mathrm{~nm}$ ) suppresses the third harmonic radiation.

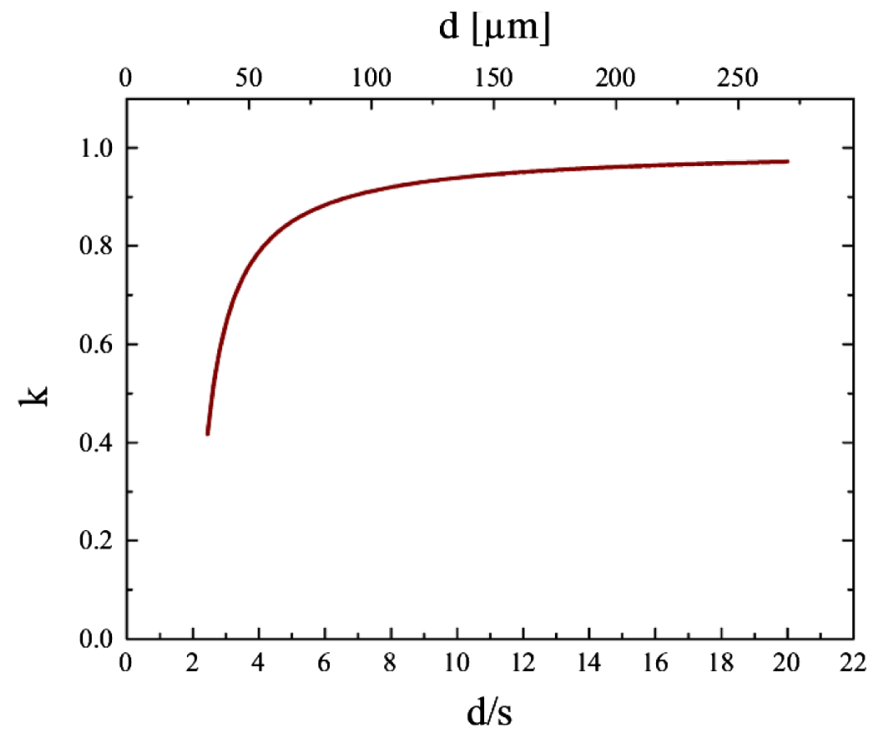

FIG. 3. Dependence of the modulation transfer function on the ratio between the fringe width $d$ of the interference pattern and the pixel size $s$ of the CCD camera.
In the experiments presented here, the electron bunches are accelerated by superconducting cavities to $442 \mathrm{MeV}$ for SASE radiation at $\lambda=32 \mathrm{~nm}$, to $511 \mathrm{MeV}$ for $\lambda=24 \mathrm{~nm}$, and up to $890 \mathrm{MeV}$ for $\lambda=8 \mathrm{~nm}$ radiation. For the SASE process the electron bunches are compressed to a short spike with a peak current of $I=(1-2.5) \mathrm{kA}$ followed by a longer tail. Undulators with an undulator period of $\lambda_{u}=$ $2.73 \mathrm{~cm}$, a length of $l=27 \mathrm{~m}, 989$ elements, and an undulator parameter of $K=1.18$ are installed. Other essential operating parameters of FLASH can be found in $[1,17]$.

\section{METHODS}

Coherence is a property of electromagnetic waves with all partial waves in phase. This property enables the waves to generate stationary interferences. A profound description of the coherence properties of light is given in terms of statistical optics. Here the second-order correlation functions play a prominent role, since for most practical cases of SASE FEL radiation they sufficiently describe the statistical properties of an electromagnetic wave field $\mathbf{E}(\mathbf{r}, \mathrm{t})$.

The intensity of the interfering beams passing the autocorrelator (see Fig. 1) at the detector is then given by the ensemble average,

$$
I\left(x_{d}, \tau\right)=\left\langle\left|\mathbf{E}_{\mathbf{1}}\left(x_{d}, t\right)+\mathbf{E}_{\mathbf{2}}\left(x_{d}, t+\tau\right)\right|^{2}\right\rangle,
$$

where $x_{d}$ is the position in the detector plane [see Fig. 4(b)], and

$$
\mathbf{E}_{\mathbf{1}}\left(x_{d}, t\right)=\mathbf{E}_{\mathbf{0 1}}\left(x_{d}, t\right) e^{i k z}
$$

and

$$
\mathbf{E}_{\mathbf{2}}\left(x_{d}, t+\tau\right)=\mathbf{E}_{\mathbf{0 2}}\left(x_{d}, t+\tau\right) e^{i\left(k z+k \alpha x_{d}\right)}
$$

are the amplitudes of the two beams passing the different arms of the autocorrelator. The additional phase in Eq. (3b), $k \alpha x_{d}$, accounts for the slightly different angle of incidence of the second beam, see Fig. 2. This angle is given by
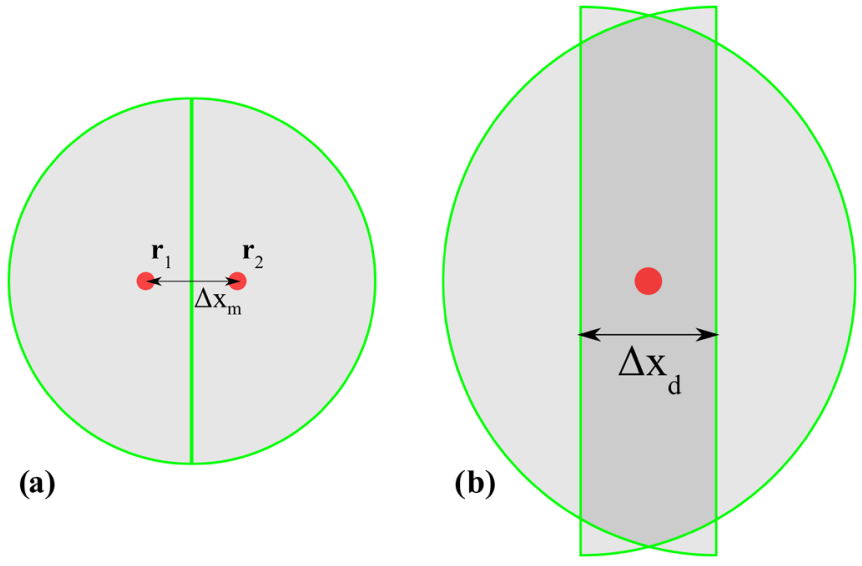

FIG. 4. (a) The beam at the splitting mirror and (b) at the detector with an overlap $\Delta x_{d}$. Because of the propagation distance of $L_{d}=20 \mathrm{~m}$ the beam appears larger here. 


$$
\alpha \approx \tan \alpha=\frac{D+\Delta x_{d}}{L_{d}},
$$

where $\Delta x_{d}$ denotes the width of the beam overlap, $D$ the separation of the two beams at the exit of the autocorrelator, and $L_{d}$ the distance between the autocorrelator and the CCD camera. In Fig. 4 the geometrical quantities discussed are illustrated. Figure 4(a) shows the distance $\Delta x_{m}$ of two points of the FEL beam on the splitting mirror of the autocorrelator. The intensity on the detector is

$$
\begin{aligned}
I\left(x_{d}, \tau\right)= & {\left[I_{1}\left(x_{d}\right)+I_{2}\left(x_{d}\right)\right] } \\
& +2 \sqrt{I_{1}\left(x_{d}\right) I_{2}\left(x_{d}\right)}\left|\gamma_{12}(\tau)\right| \cos \left[k \alpha x_{d}+\alpha_{12}(\tau)\right],
\end{aligned}
$$

where $I_{1,2}\left(x_{d}\right)=\left\langle E_{1,2}^{2}\left(x_{d}, t\right)\right\rangle, \gamma_{12}(\tau)$ is the complex degree of coherence [Eq. (7)], and $\alpha_{12}\left(x_{d}, \tau\right)$ is the phase of the complex degree of coherence. The first term accounts for the intensities of the two partial beams. The second term introduces a modulation via the cosine function. Since $\gamma_{12}(\tau)$ decreases with increasing time delay $\tau$ as well as with increasing spatial overlap, this term vanishes for long delays and for large distances between the points $\mathbf{r}_{\mathbf{1}}$ and $\mathbf{r}_{\mathbf{2}}$. The complex degree of mutual coherence $\gamma_{12}(\tau)$ is given as the normalized mutual coherence function $\Gamma\left(\mathbf{r}_{1}, \mathbf{r}_{2}, \tau\right)$ that describes the correlations of an electromagnetic wave field $\mathbf{E}(\mathbf{r}, t) . \Gamma\left(\mathbf{r}_{1}, \mathbf{r}_{2}, \tau\right)$ is defined by the correlation function of the wave field $\mathbf{E}(\mathbf{r}, t)$ at two positions $\mathbf{r}_{1}$ and $\mathbf{r}_{2}$ at two different times $t$ and $(t+\tau)$ [18]:

$$
\Gamma\left(\mathbf{r}_{1}, \mathbf{r}_{2}, \tau\right)=\left\langle\mathbf{E}\left(\mathbf{r}_{1}, \mathbf{t}\right) \mathbf{E}^{*}\left(\mathbf{r}_{2}, \mathbf{t}+\tau\right)\right\rangle .
$$

The absolute value of the normalized correlation function

$$
\left|\gamma_{12}(\tau)\right|=\left|\frac{\Gamma\left(\mathbf{r}_{1}, \mathbf{r}_{2}, \tau\right)}{\sqrt{\Gamma\left(\mathbf{r}_{1}, \mathbf{r}_{1}, 0\right) \Gamma\left(\mathbf{r}_{2}, \mathbf{r}_{2}, 0\right)}}\right|
$$

can be measured via the visibility $V$ of the interference fringes of two interfering partial beams. This visibility $V$ is given by the maximum and adjacent minimum intensity of the interference pattern,

$$
V=\frac{I_{\max }-I_{\min }}{I_{\max }+I_{\min }}=\left|\gamma_{12}(\tau)\right|\left\{2 \sqrt{I_{1} I_{2}} /\left(I_{1}+I_{2}\right)\right\},
$$

where $I_{1}$ and $I_{2}$ are the intensities of the interfering partial beams and $I_{\max }$ and $I_{\min }$ are the maximum and minimum intensities of the interference fringes.

\section{A. Temporal coherence}

The temporal coherence is described by $\left|\gamma_{12}(\tau)\right|$ as a function of $\tau$ for fixed points $\mathbf{r}_{1}$ and $\mathbf{r}_{2}$. The coherence time $\tau_{c}$ can be defined as the half width at half maximum (HWHM) of $\left|\gamma_{12}(\tau)\right|$. A more general definition for an arbitrary function is given by the rms value [18]

$$
\tau_{c, \mathrm{rms}}=\int_{-\infty}^{\infty}\left|\gamma_{12}(\tau)\right|^{2} d \tau
$$

For a Gaussian function this results in a coherence time of $\tau_{c, \mathrm{rms}} \approx 0.85 \tau_{c}$.

\section{B. Spatial coherence}

The magnitude of $\left|\gamma_{12}(\tau)\right|$ as a function of the distance between $\mathbf{r}_{\mathbf{1}}$ and $\mathbf{r}_{\mathbf{1}}$ for a constant $\tau$ describes the spatial coherence of the field. The width of this function is called the transverse coherence length $\xi_{x, y}$ in horizontal $(x)$ and vertical $(y)$ directions. In analogy to the coherence time of the light pulse (9) the degree of transverse coherence $\zeta$ is defined as [11,19]

$$
\zeta=\frac{\iint\left|\gamma\left(\mathbf{r}_{1}, \mathbf{r}_{2}\right)^{2}\right|\left\langle I\left(\mathbf{r}_{1}\right)\right\rangle\left\langle I\left(\mathbf{r}_{2}\right)\right\rangle d \mathbf{r}_{1} d \mathbf{r}_{2}}{\left[\int\langle I(\mathbf{r})\rangle d \mathbf{r}\right]^{2}} .
$$

From the mutual coherence function $\Gamma\left(\mathbf{r}_{1}, \mathbf{r}_{2}, \tau\right)$ [Eq. (6)], the cross spectral density $W\left(\mathbf{r}_{1}, \mathbf{r}_{2}, \omega\right)$ is defined as

$$
W\left(\mathbf{r}_{1}, \mathbf{r}_{2}, \omega\right)=\int_{-\infty}^{\infty} \Gamma\left(\mathbf{r}_{1}, \mathbf{r}_{2}, \tau\right) e^{i \omega \tau} d \tau .
$$

From this expression one can readily recognize that it forms a Fourier pair with the mutual coherence function $\Gamma\left(\mathbf{r}_{1}, \mathbf{r}_{2}, \tau\right)$. The cross spectral density constitutes a measure between the spectral amplitudes of any particular frequency component $\omega$ of the electromagnetic field at the spatial points $\mathbf{r}_{\mathbf{1}}$ and $\mathbf{r}_{\mathbf{2}}$. When the cross spectral density $W\left(\mathbf{r}_{1}, \mathbf{r}_{2}, \omega\right)$ is evaluated for one point $\mathbf{r}_{1}=\mathbf{r}_{2}=\mathbf{r}$ it equals the power spectrum $S(\mathbf{r}, \omega)$ of the light. Analogous to the mutual coherence function $\Gamma\left(\mathbf{r}_{1}, \mathbf{r}_{2}, \tau\right)$ also the cross spectral density $W\left(\mathbf{r}_{1}, \mathbf{r}_{2}, \omega\right)$ can be normalized:

$$
\mu\left(\mathbf{r}_{1}, \mathbf{r}_{1}, \omega\right)=\frac{W\left(\mathbf{r}_{1}, \mathbf{r}_{2}, \omega\right)}{\sqrt{S\left(\mathbf{r}_{1}, \omega\right)} \sqrt{S\left(\mathbf{r}_{2}, \omega\right)}} .
$$

For a stationary field the cross spectral density $W\left(\mathbf{r}_{1}, \mathbf{r}_{2}, \omega\right)$ can be represented by a sum of independent coherent modes [20],

$$
W\left(\mathbf{r}_{1}, \mathbf{r}_{2}, \omega\right)=\sum_{j=0}^{n} \beta_{j} \psi_{j}^{*}\left(\mathbf{r}_{1}\right) \psi_{j}\left(\mathbf{r}_{2}\right),
$$

where $\beta_{j}$ and $\psi_{j}$ are the eigenvalues and eigenfunctions of the Fredholm integral equation,

$$
\int W\left(\mathbf{r}_{1}, \mathbf{r}_{2}\right) \psi_{j}\left(\mathbf{r}_{1}\right) d \mathbf{r}_{1}=\beta_{j} \psi_{j}\left(\mathbf{r}_{2}\right) .
$$

If the intensity distribution $I(\mathbf{r})$ and the complex coherence factor $|\mu(\Delta x)|$ are both Gaussian, the cross spectral density $W\left(\mathbf{r}_{1}, \mathbf{r}_{2}, \omega\right)$ can be represented by the well-known GaussHermite polynomials. If the intensity profile and the complex coherence factor are not Gaussian, the modes have to be found by solving the Fredholm integral equation (14) with the corresponding cross spectral density and will differ from Gaussian-Hermite modes.

To determine the transverse coherence of the FLASH pulses at the entrance of the autocorrelator, the divergence 
of the beam has to be taken into account. At the large distance from the undulator source of $70 \mathrm{~m}$, the beam size and the transverse coherence length are considered to change approximately linearly with the propagation distance. The overlap of the two partial beams on the CCD camera, $\Delta x_{d}$ [see Fig. 4(b)], corresponds to a separation at the splitting mirror, $\Delta x_{m}$, via

$$
\Delta x_{m}=\frac{L_{s}}{L} \Delta x_{d}
$$

where $L=L_{s}+L_{\mathrm{AC}}+L_{d}$ is the total distance between the source and the detector. Here $L_{s}$ denotes the distance between the source and the autocorrelator, $L_{\mathrm{AC}}$ the length of the autocorrelator, and $L_{d}$ the already mentioned distance between the autocorrelator and the detector. A measurement of the visibility at different overlap widths $\Delta x_{d}$ corresponds to double pinhole measurements with pinhole separations of $\Delta x_{m}$. From Eq. (15) and

$$
x_{m}=\frac{L_{s}}{L} x_{d},
$$

the dimensions of the beam on the splitting mirror can be retrieved from the dimensions measured on the CCD camera.

\section{COHERENCE PROPERTIES OF SASE FEL RADIATION}

\section{A. Temporal coherence}

FLASH is a free-electron laser based on self-amplified spontaneous emission (SASE FEL). It emits coherent XUV radiation during a single pass of an electron bunch through a sufficiently long undulator. According to FEL theory $[7,8,10,11,21]$, the emission of spontaneous undulator radiation is a stochastic process. As a consequence SASE FEL radiation, starting from shot noise, has the properties of chaotic light [21]. The process of radiation emission is initiated in the first part of the undulator by a density modulation of the electron bunch with a period length close to the resonance wavelength $\lambda$ of the undulator,

$$
\lambda=\lambda_{u}\left(1+\frac{K^{2}}{2}\right) /\left(2 \gamma^{2}\right)
$$

Here $\lambda_{u}$ denotes the period of the undulator, $K$ is the undulator parameter (at FLASH the undulator parameter is $K=1.18)$, and $\gamma=\left[1-(v / c)^{2}\right]^{-1 / 2}$ is the relativistic factor. The oscillating electrons interact with the radiation produced by themselves. The electric force of the light wave causes an energy modulation and subsequently a longitudinal density modulation in the electron bunch with a period similar to the resonance wavelength, thereby inducing a microbunching of the electrons. The radiation emitted by individual microbunches is phase locked and therefore adds coherently, leading to a further enhancement of the microbunching. Within this process, known as self-amplified spontaneous emission (SASE), the intensity of the FEL pulse develops exponentially with the undulator length. In the saturation regime almost all electrons within the coherence length $L_{c}$ radiate in phase producing coherent radiation. The coherence length $L_{c}$ is approximately given by [22]

$$
L_{c} \approx \lambda L_{g} / \lambda_{u},
$$

where $L_{g}$ is the gain length in the undulator,

$$
L_{g}=\lambda_{u} /(4 \pi \rho) \text {. }
$$

Here $\rho$ is the FEL parameter $[7,23,24]$

$$
\rho=\frac{1}{2 \gamma}\left[\frac{I}{I_{A}}\left(\frac{A_{J J} K \lambda_{u}}{2 \pi \sigma_{\perp}}\right)^{2}\right]^{1 / 3} .
$$

In this expression $I$ describes the peak beam current, $I_{A}$ the Alfvén current, and $\sigma_{\perp}$ the rms transverse size of the electron bunch. The coupling factor is $A_{J J}=1$ for a helical undulator and $A_{J J}=\left|J_{0}(Q)-J_{1}(Q)\right|$ for a planar undulator $\left(A_{J J}=0.84\right.$ at FLASH), where $Q=K^{2} /\left[2\left(1+K^{2}\right)\right]$ and $J_{0}$ and $J_{1}$ are Bessel functions of the first kind. At FLASH different wavelengths are generated by tuning the energy of the electron bunch. Hence, for constant peak current the FEL parameter $\rho$ basically is inversely proportional to the relativistic factor,

$$
\rho \propto \gamma^{-1},
$$

if the other parameters are assumed to be constant for different wavelengths. It should be noted that the transverse beam size $\sigma_{\perp}$ and the peak bunch current $I$ are susceptible to changes due to the daily optimization of the linear accelerator. However, assuming for a moment constant values in these parameters, then together with Eqs. (17)-(19) a nonlinear dependence of the coherence length $L_{c}$ on the wavelength $\lambda$ of the FEL radiation becomes evident:

$$
L_{c} \propto \lambda^{1 / 2} \text { or } \tau_{c} \propto \lambda^{1 / 2} .
$$

Because of its nature and thus arising from noise, the radiation of SASE FELs consists of independently radiating transverse and longitudinal modes. In the time domain the radiation is emitted in short spikes (temporal modes) with duration $\tau_{c}$ and with a random phase relationship between the spikes [25]. Time domain and spectral domain of each subpulse are related to each other via a Fourier transformation which leads to narrow spikes (spectral modes) within the bandwidth of the undulator in the spectral domain, too. In the linear autocorrelation experiments presented here these independent modes can interact with each other at longer time delays as a cross correlation. This behavior causes a slower decay of the visibility at longer time delays. Hence, calculating the coherence time with Eq. (9) may yield higher values compared to the HWHM of a Gaussian fit. For a flattop electron bunch with a time duration of $T_{\text {bunch }}$, the number of longitudinal modes $M_{l}$ can be estimated via 


$$
M_{l}=\frac{T_{\text {bunch }}}{\tau_{c}}
$$

However, in the experiments presented here FLASH was operated in the femtosecond mode, where it cannot be assumed that the bunch follows a flattop time profile. Instead, the electron bunch is compressed to a short spike with a peak current of $I=2.5 \mathrm{kA}$ which is followed by a longer tail [1]. Thus, an estimation of the number of modes via Eq. (23) will not be accurate.

\section{B. Spatial coherence}

One of the most outstanding features of SASE FELs is that a high degree of spatial coherence can be achieved. According to 3D FEL theory [8], a large number $M$ of transverse radiation modes is excited when the electron beam enters the undulator, because the fluctuations of the current density in the electron bunch are uncorrelated not only in time but also in space. Since these different modes have different spatial overlap with the electron beam, the amplification they experience is different, too. Therefore the number of modes decreases during the amplification process. As the fundamental mode has the best spatial overlap with the electron beam, the field amplitude is dominated by the fundamental mode, which theoretically contributes close to $99.9 \%$ to the radiated power [26]. In the linear regime the degree of transverse coherence [Eq. (10)] can be approximated by $\zeta=1 / M$. Transverse coherence establishes quickly at an early stage of the amplification due to the transverse mode selection. One could mistakenly deduce that the latter effect proceeds further at larger distances $z$ of amplification in the undulator and that the degree of transverse coherence therefore should approach unity exponentially. But it was predicted that at larger values of the undulator length the degree of transverse coherence approaches unity only asymptotically as $(1-\zeta) \propto 1 / z$ [8]. At this point, one should take a closer look at the spiky temporal and spectral structure of the FEL pulses. In the high-gain linear regime the radiation of the SASE FEL consists of spatial fundamental modes, however, at many different frequencies. The transverse distributions of the radiation field of spatially fundamental modes are also slightly different for different frequencies. As a result spatial interference of these longitudinal modes can occur and full transverse coherence is not achieved during the SASE process. To conclude, the interdependence between longitudinal coherence and transverse coherence is possibly accountable for the fact that full transverse coherence is not achieved even after completion of the mode selection process. When the fundamental mode reaches saturation, the higher modes are not yet saturated. Since the amplification process still proceeds with increasing undulator length, these modes can continue to grow. In consequence, the degree of transverse coherence may remain then even theoretically below $\zeta=0.9$.

\section{Temporal coherence of the odd harmonics}

In SASE FEL employing a planar undulator spontaneous emission induces microbunching at the fundamental resonance frequency of the undulator. Since the trajectory of the particle motion is not strictly sinusoidal, the emitted radiation also contains higher odd harmonics of the fundamental wavelength. The properties of the harmonic radiation are described in [9]. For the fundamental harmonic the coherence time achieves its maximal value near the saturation point and then decreases. According to [9] the longitudinal coherence of the higher harmonics evolves in three stages. First, the longitudinal coherence increases linearly with the undulator length $z$. When the process of nonlinear harmonic generation starts to dominate over spontaneous emission, the coherence time drops sharply. Before the amplification process reaches saturation at the end of the exponential regime there is a plateau where the ratio of the longitudinal coherence of the $n$th harmonic to that of the first harmonic scales with $\tau_{c} \propto 1 / \sqrt{n}$. At saturation point the coherence time falls inversely proportional to the harmonic order $\tau_{c} \propto 1 / n$.

\section{EXPERIMENTAL RESULTS}

\section{A. Temporal coherence}

For the measurement of the temporal coherence both partial beams are overlapped only in the center $\left(\Delta x_{d}=\right.$ $0.9 \mathrm{~mm}$ ) and the temporal delay between the two pulses is scanned. The temporal coherence of the FEL beam is investigated for $\lambda=32,24,13$, and $8 \mathrm{~nm}$. Figure 5 displays the observed visibility of the interference fringes (corrected for the detector MTF) versus the delay between the partial beams, thus representing the temporal coherence function of FLASH pulses at $\lambda=24 \mathrm{~nm}$ [Fig. 5(a)] and at $\lambda=$ $8 \mathrm{~nm}$ [Fig. 5(b)] fundamental wavelengths. At each time delay, the average of the visibility over ten individual pulses is shown. Error bars give the standard deviation of this average. At zero delay the visibility reaches an averaged maximum of $V=0.89$ at $\lambda=24 \mathrm{~nm}$ fundamental wavelength, and $V=0.85$ at $\lambda=8 \mathrm{~nm}$. Occasionally also complete modulation, i.e., $V=1.0$, is observed. The reason why the visibility does not reach unity is an insufficient spatial coherence, see below and discussion above. The average temporal coherence function of the pulses reveals two different time scales. First, the visibility rapidly decreases showing a Gaussian decay in the central part. For time delays larger than $10 \mathrm{fs}$, the decrease of the visibility slows down, revealing a weaker correlation also for longer time scales. This behavior is a consequence of the multispike structure of the spectra of the FEL pulses which has been discussed before and in Ref. [14]. The central part of the data is fitted with a Gaussian function from which the coherence time can be extracted. For this central part coherence times of $\tau_{c}=(6 \pm 0.5) \mathrm{fs}$ and $\tau_{c}=(2.9 \pm 0.5) \mathrm{fs}$ are observed for 24 and $8 \mathrm{~nm}$ radiation, respectively. 

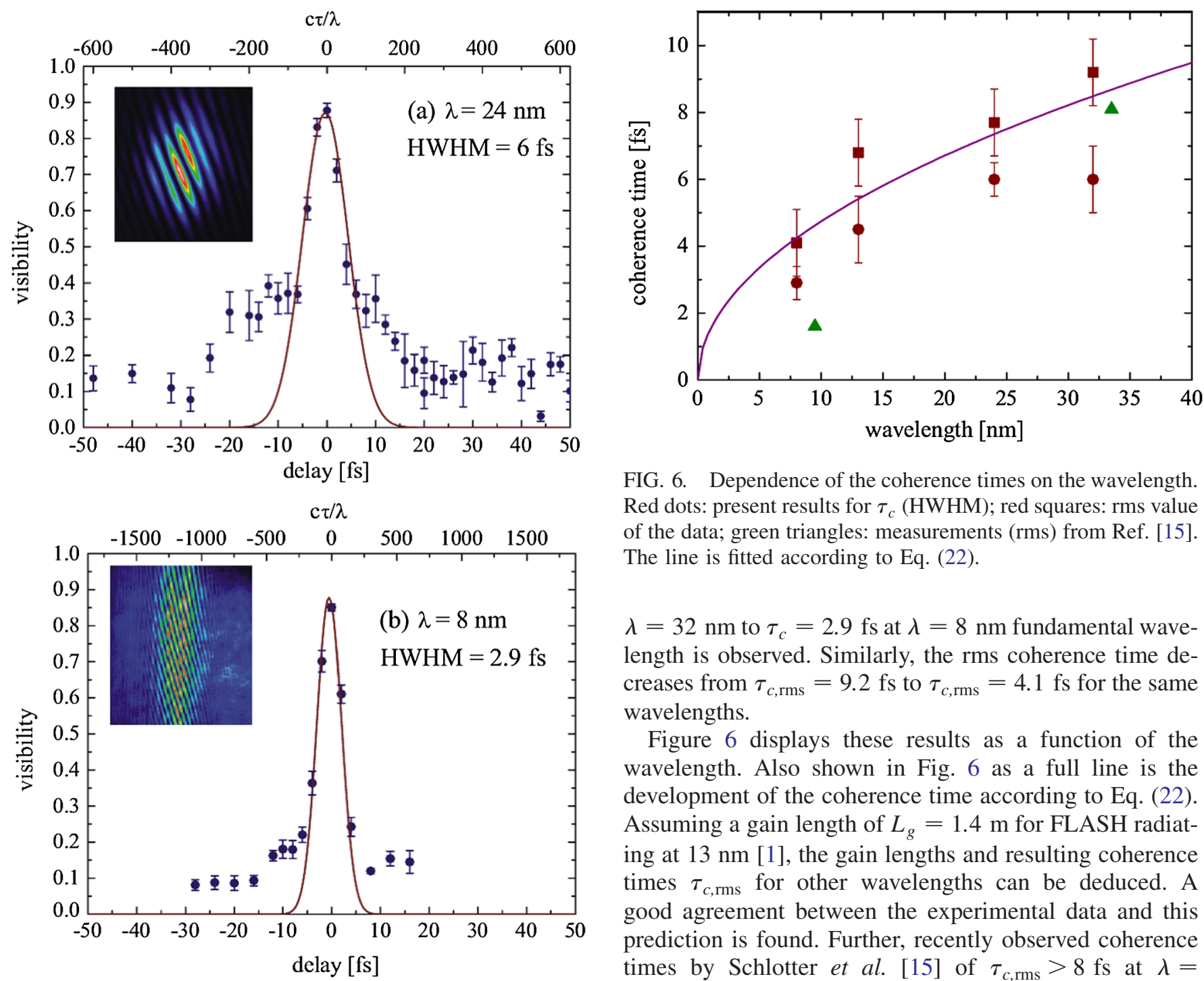

FIG. 6. Dependence of the coherence times on the wavelength. Red dots: present results for $\tau_{c}$ (HWHM); red squares: rms value of the data; green triangles: measurements (rms) from Ref. [15]. The line is fitted according to Eq. (22).

$\lambda=32 \mathrm{~nm}$ to $\tau_{c}=2.9 \mathrm{fs}$ at $\lambda=8 \mathrm{~nm}$ fundamental wavelength is observed. Similarly, the rms coherence time decreases from $\tau_{c, \text { rms }}=9.2$ fs to $\tau_{c, \text { rms }}=4.1$ fs for the same wavelengths.

Figure 6 displays these results as a function of the wavelength. Also shown in Fig. 6 as a full line is the development of the coherence time according to Eq. (22). Assuming a gain length of $L_{g}=1.4 \mathrm{~m}$ for FLASH radiating at $13 \mathrm{~nm}$ [1], the gain lengths and resulting coherence times $\tau_{c, \text { rms }}$ for other wavelengths can be deduced. A good agreement between the experimental data and this prediction is found. Further, recently observed coherence times by Schlotter et al. [15] of $\tau_{c \text {,rms }}>8$ fs at $\lambda=$ $33.2 \mathrm{~nm}$ and $\tau_{c, \text { rms }}=1.6 \mathrm{fs}$ at $\lambda=9.6 \mathrm{~nm}$ are shown for completeness. Also these data generally agree well with the results obtained in this work. It should be mentioned that the data discussed in this work are averages of the visibility of randomly selected FLASH pulses, operating in the femtosecond single bunch mode. The deduced coherence times are based on the assumption of stable operating conditions of FLASH over the course of data taking of about $1.5 \mathrm{~h}$. Evidently a single shot measurement of the temporal coherence could serve to enhance the understanding of the SASE process even better.

TABLE I. The coherence time $\tau_{c}$ at different wavelength. $\tau_{c}$ is given as the HWHM of the Gaussian functions shown in Fig. 5; $\tau_{c, \text { rms }}$ is calculated with Eq. (9).

\begin{tabular}{lccl}
\hline \hline Wavelength & Pulse energy $[\mu \mathrm{J}]$ & $\tau_{c}[\mathrm{fs}]$ & $\tau_{c, \mathrm{rms}}[\mathrm{fs}]$ \\
\hline $32 \mathrm{~nm}$ fundamental & 25 & $6 \pm 0.5$ & $9.2 \pm 0.5$ \\
$24 \mathrm{~nm}$ fundamental & $25-30$ & $6 \pm 0.5$ & $7.7 \pm 0.5$ \\
$13 \mathrm{~nm}$ fundamental & $34-42$ & $4.5 \pm 1$ & $6.8 \pm 1$ \\
$8 \mathrm{~nm}$ fundamental & 3 & $2.9 \pm 0.5$ & $4.1 \pm 0.5$ \\
$8 \mathrm{~nm}$ 3rd & $0.16-0.19$ & $2.4 \pm 0.5$ & $3.6 \pm 0.5$ \\
\hline \hline
\end{tabular}

\section{B. Spatial coherence}

The spatial coherence of the FLASH pulses was measured at $\lambda=24 \mathrm{~nm}$ and $\lambda=8 \mathrm{~nm}$. During all measurements a $3 \mathrm{~mm}$ aperture was deployed $20 \mathrm{~m}$ downstream from the undulator exit, $50 \mathrm{~m}$ in front of the autocorrelator. The time delay $\tau$ was set to zero for this measurements. By increasing the spatial overlap of the two partial beams on the CCD camera, the distance between two points of the spatial beam profile interfering with each other is increased, 

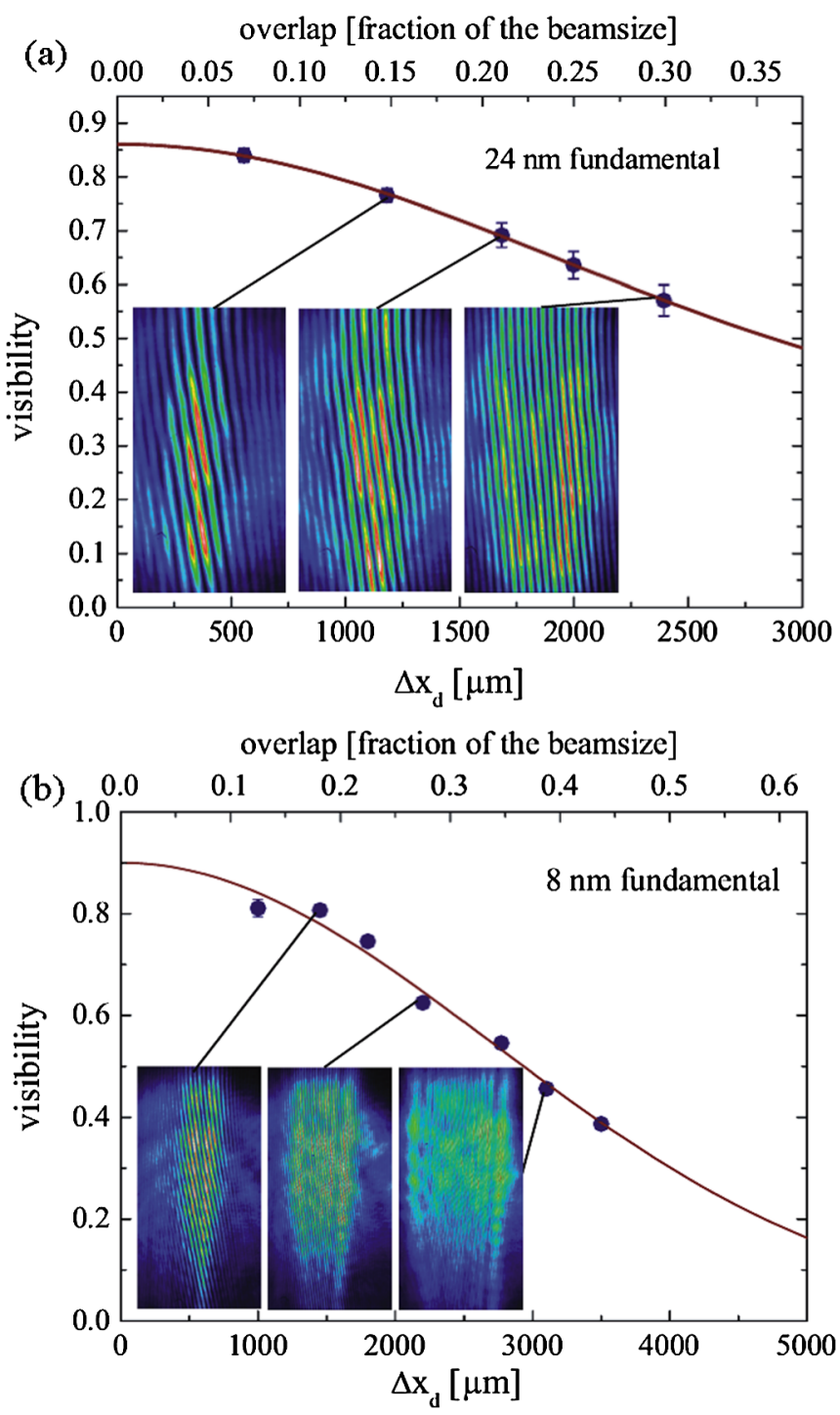

FIG. 7. Spatial coherence at (a) $24 \mathrm{~nm}$ and (b) $8 \mathrm{~nm}$ fundamental wavelength. The insets show typical single shot interference pattern at different overlaps. The solid line is a Gaussian fit.

see Fig. 4. Figure 7 shows the average visibility as a function of the overlap $\Delta x_{d}$ at the detector position and as a fraction of the beam size at the detector (upper scale). Again averages of ten individual pulses at each overlap are displayed. The insets show typical individual interferograms at certain overlaps. The visibility decreases with increasing $\Delta x_{d}$ as expected. The data are fitted well with a Gaussian function. The measured HWHM and rms values of $\xi_{d}$ at the detector are shown in Table II. At the detector the visibility diminishes with a rms value of $\xi_{d}=3.0 \mathrm{~mm}$ at $\lambda=24 \mathrm{~nm}$ (corresponding to a HWHM of $3.52 \mathrm{~mm}$ ) and $\xi_{d}=2.7 \mathrm{~mm}$ at $\lambda=8 \mathrm{~nm}$ fundamental wavelength (corresponding to a HWHM of $3.2 \mathrm{~mm}$ ). Employing Eq. (16) this yields a transverse coherence length (rms) at $\lambda=$ $24 \mathrm{~nm}$ of $\xi_{\text {in }}=2.3 \mathrm{~mm}$ (corresponding to a HWHM of $2.7 \mathrm{~mm}$ ) at the entrance of the autocorrelator. Here the beam
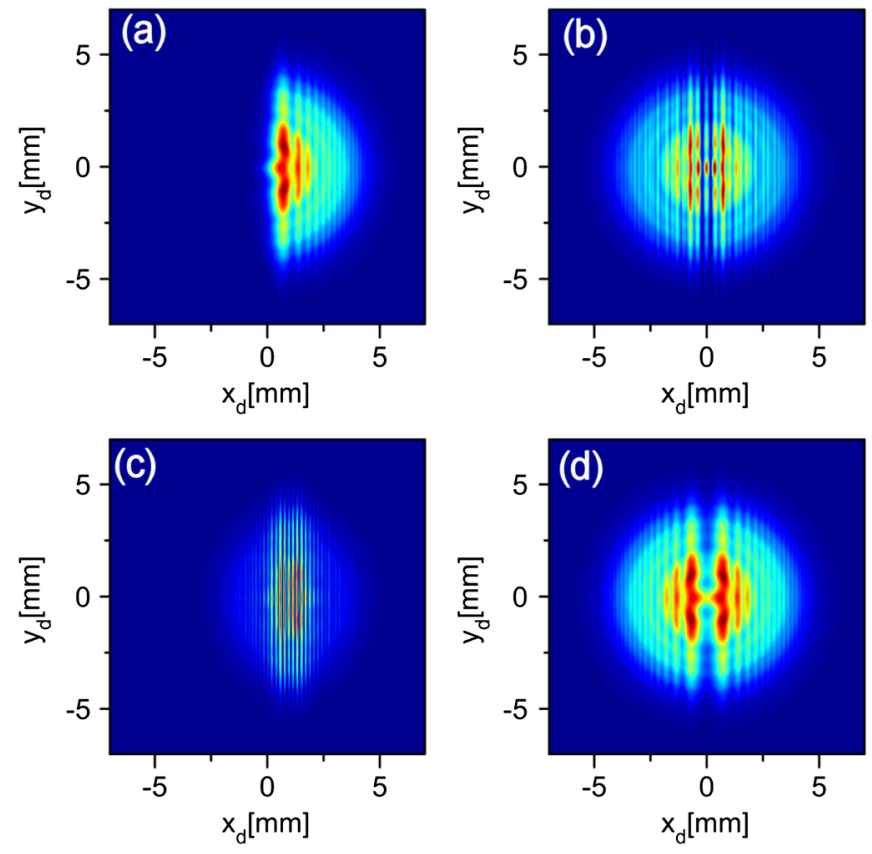

FIG. 8. Simulations of the beam profile at the detector. (a) The beam transmitted through one arm of the autocorrelator. (b) Zero overlap of two temporally and spatially coherent beams $\left[\gamma_{12}(\tau)=1\right]$. (c) A $2 \mathrm{~mm}$ overlap of two temporally and spatially coherent beams $\left[\gamma_{12}(\tau)=1\right]$. (d) Zero overlap of two temporally incoherent beams $\left[\gamma_{12}(\tau)=0\right]$.

size (rms) is $\sigma_{\text {in }}=2.5 \mathrm{~mm}$ (corresponding to a FWHM of $5.88 \mathrm{~mm}$ ). These values yield a transverse degree of coherence of $\zeta=0.42$.

The mentioned pinhole may cut higher modes and thus gives a higher degree of spatial coherence. Thus the values given here do not necessarily describe the coherence properties of the source, but the coherence properties at the experimental station. Although assuming simply a Gaussian propagation of the beam, from the beam diameter at the entrance of the autocorrelator of $\sigma_{\text {in }}=2.5 \mathrm{~mm}$ one deduces a beam diameter $\left(1 / e^{2}\right)$ of $2.9 \mathrm{~mm}$ at the position of the $3 \mathrm{~mm}$ pinhole. Figure 8 shows simulations of the beam profile at the detector taking into account both diffraction from the pinhole and from the edge of the beam splitting mirror. It is evident that the results of Fig. 8(c) resemble the experimental results best. The experimental coherence data are analyzed in the frame of the Gaussian Schell model (GSM) by means of the mode decomposition of the correlation function $[16,19]$ in order to estimate the number of transverse modes contributing to the beam profile. The GSM is a good approximation in our case, since the measured modulus of the degree of coherence is well approximated by Gaussian functions (see Fig. 7) and the intensity profile observed in our measurement can also be considered as Gaussian (apart from the fringes due to edge scattering at the split mirror of the autocorrelator). The modulus of the degree of coherence $|\mu(\Delta x)|$ and the corresponding intensity profile of the FLASH pulse at the 


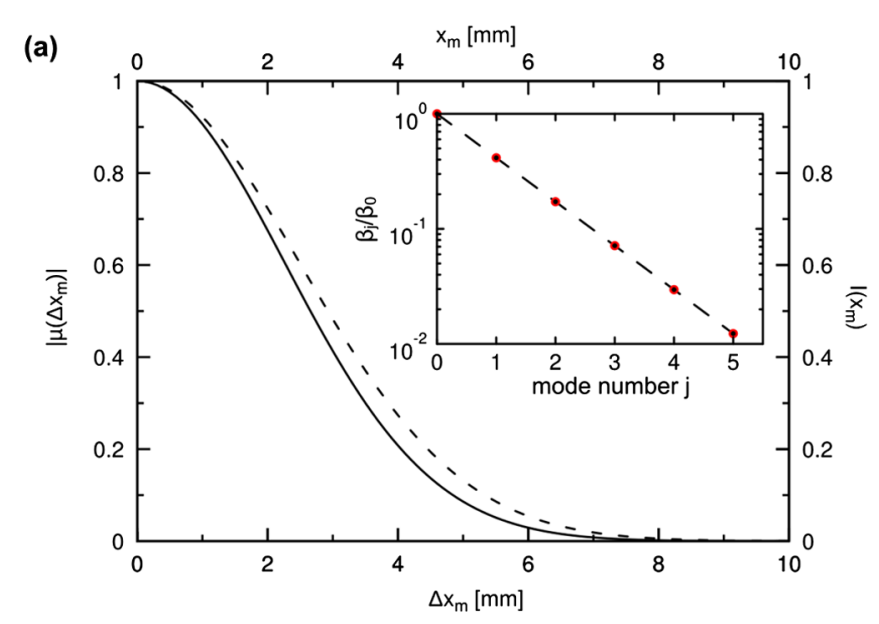

(b)
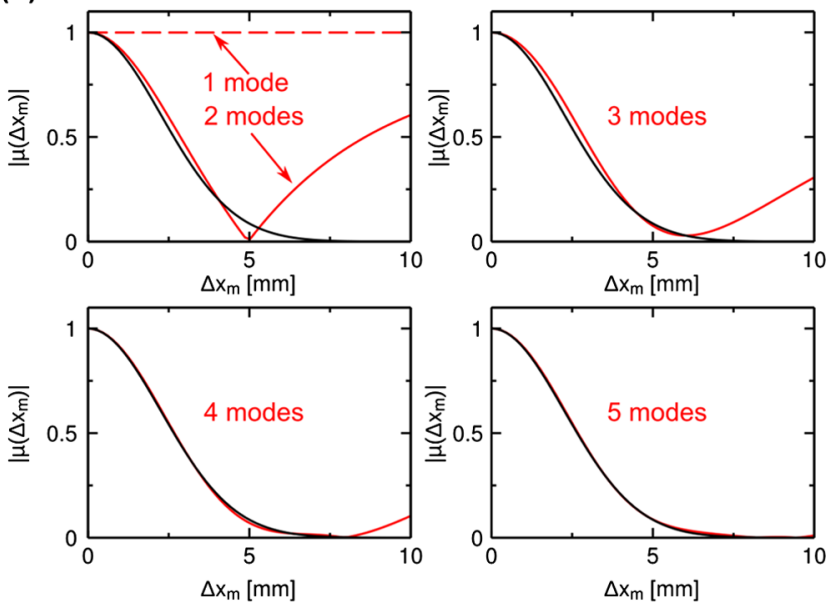

FIG. 9. (a) The modulus of the complex coherence factor $|\mu(\Delta x)|$ at the entrance of the autocorrelator for FLASH pulses at $\lambda=24 \mathrm{~nm}$ (solid line, lower abscissa). The dashed line represents the intensity distribution $I(x)$ (upper abscissa). The inset shows the ratio $\beta_{j} / \beta_{0}$ of the eigenvalue $\beta_{j}$ to the lowest order eigenvalue $\beta_{0}$ as a function of the mode number $j$. (b) The contribution of the transverse modes to the complex coherence factor $\left|\mu\left(\Delta x_{m}\right)\right|$.

entrance of the autocorrelator are shown in Fig. 9(a) as a function of the spatial beam overlap $\Delta x_{m}$. The contribution $\beta_{j}$ of different modes $\psi_{j}$ to the total radiated intensity is shown in Fig. 9(b) in a logarithmic scale. Evidently six modes with contributions of greater than $1 \%$ have to be considered. It is seen in Fig. 9 that these six modes sufficiently describe also the coherence properties of the FLASH pulses. It might now be conceived that this result significantly differs from the theoretical expectation of approximately 1-2 modes contributing to the radiation at the end of the linear regime in the undulator. However, one has to recognize that in the saturation regime the degree of transverse coherence decreases again due to the fact that higher modes continue to grow and catch up in intensity. Furthermore, in the theoretical description idealized assumptions have to be made, e.g., the transverse distribution

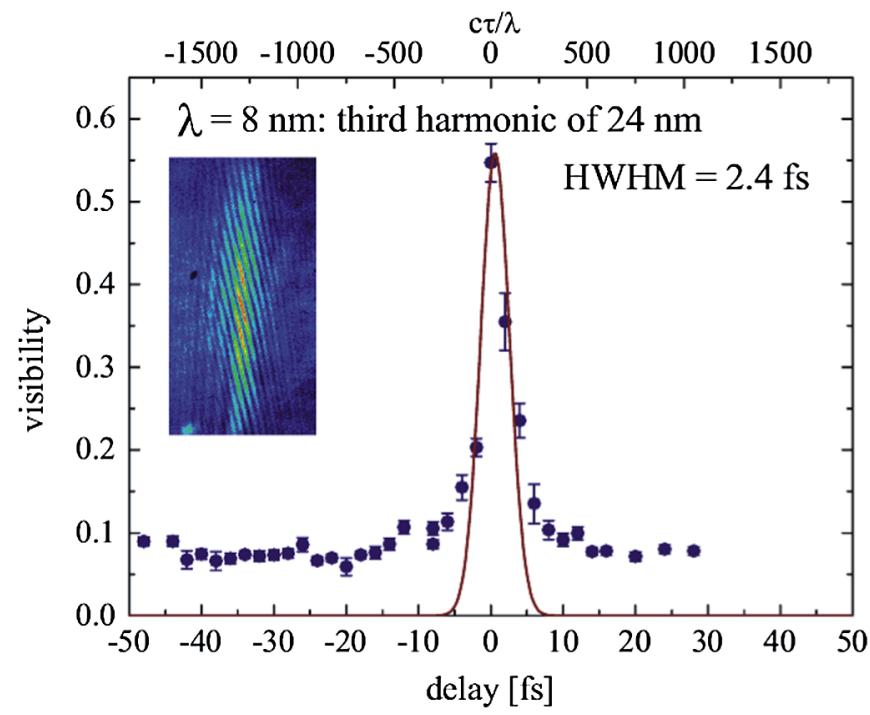

FIG. 10. Visibility as a function of pulse delay for $\lambda=8 \mathrm{~nm}$ as the third harmonic of $24 \mathrm{~nm}$ fundamental wavelength. The inset shows interference fringes at zero delay with $V=0.71$. A coherence time of $\tau_{c}=2.4 \mathrm{fs}$ is obtained in this case. The solid line is a Gaussian fit.

of the electron beam current is assumed to be Gaussian $[8,26]$. The description of the beam by the GSM is an approximation that can be applied to calculate the propagation of the beam. It does not give information about the generation of the FEL pulse in the SASE process.

\section{Coherence properties of the third harmonic}

In addition to the measurements at fundamental wavelengths, the temporal and spatial coherence at $\lambda=8 \mathrm{~nm}$ as third harmonic of $\lambda=24 \mathrm{~nm}$ was investigated. Figure 10 shows the visibility averaged over ten single pulses as a function of the delay between the partial beams. A maximum averaged visibility of $V=0.55$ is observed, with individual pulses reaching up to $V=0.71$. The inset shows a typical interferogram at $\Delta \tau=0$ fs with $V=0.71$. The data shows a coherence time of $\tau_{c}=(2.4 \pm 0.5) \mathrm{fs}$ (HWHM), as compared to $\tau_{c}=(6 \pm 0.5)$ fs for the fundamental at $\lambda=24 \mathrm{~nm}$. For the rms temporal coherence a value of $\tau_{c, \mathrm{rms}}=(3.7 \pm 0.5)$ fs is deduced. In comparison, for $8 \mathrm{~nm}$ fundamental wavelength a slightly higher coherence time of $\tau_{c}=(2.9 \pm 0.5) \mathrm{fs}$ is found. This result is in good agreement with estimations from FEL theory [9], which states that at saturation point the temporal coherence should scale with $1 / n$, where $n$ is the harmonic number. Before saturation, in the exponential gain regime, the coherence time scales with $1 / \sqrt{n}$. Figure 11 shows for the third harmonic the averaged visibility as a function of the spatial beam overlap $\Delta x_{d}$ at the detector, or as a fraction of the beam profile. The visibility decreases with increasing $\Delta x_{d}$ as expected. A Gaussian fit to the visibility reveals a transverse coherence length (rms) of $\xi_{d}=$ $2.3 \mathrm{~mm}$. The absolute value of the visibility is slightly 


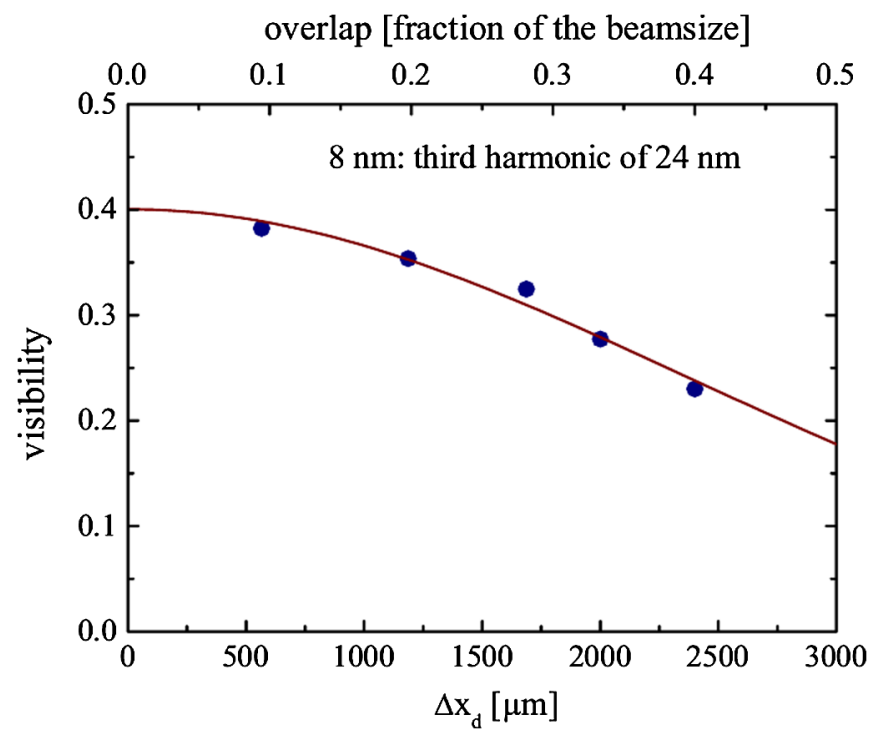

FIG. 11. Spatial coherence of the third harmonic at $\lambda=8 \mathrm{~nm}$.

reduced due to the circumstance that this measurement was taken at $\tau=2$ fs instead of $\tau=0$ fs.

Employing special filters which transmit in the upper part the fundamental and in the lower part the third harmonic radiation allows one to measure the visibility for both simultaneously and therefore to investigate correlations between both for the same pulse and thus the same electron bunch. In the present case this is achieved by a combination of $\mathrm{Zr}$ and $\mathrm{Al}$ filters, which separate the $8 \mathrm{~nm}$ third harmonic and the $24 \mathrm{~nm}$ fundamental. Figure 12 shows in the inset such a typical recording. In this case the visibility of the fundamental amounts to $V(24 \mathrm{~nm})=0.78$ and that of the

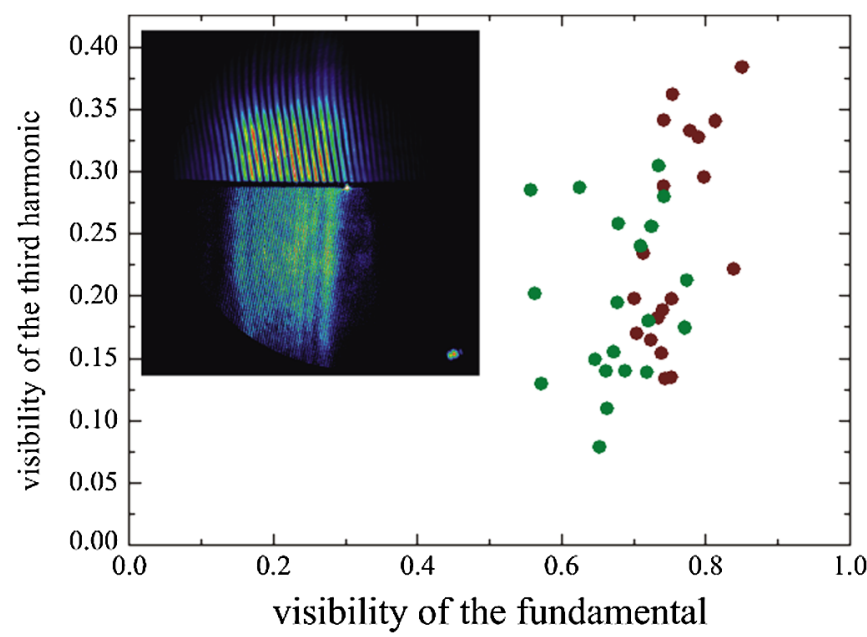

FIG. 12. Correlation between $24 \mathrm{~nm}$ fundamental wavelength and the third harmonic radiation. The inset shows interference fringes simultaneously measured for $24 \mathrm{~nm}$ (upper) and $8 \mathrm{~nm}$ (lower) by means of $\mathrm{Al} / \mathrm{Zr}$ filters. Green dots are taken at $\Delta t=$ $+2.5 \mathrm{fs}$, red dots at $\Delta t=-1.5 \mathrm{fs}$.
TABLE II. The transverse coherence at different wavelength. Measured HWHM and rms values of $\xi_{d}$ at the detector position.

\begin{tabular}{lcc}
\hline \hline Wavelength & $\xi_{d}[\mathrm{~mm}](\mathrm{rms})$ & $\xi_{d}[\mathrm{~mm}](\mathrm{HWHM})$ \\
\hline 24 nm fundamental & 3.0 & 3.52 \\
$8 \mathrm{~nm}$ fundamental & 2.7 & 3.2 \\
$8 \mathrm{~nm}$ 3rd & 2.3 & 2.7 \\
\hline \hline
\end{tabular}

third harmonic to $V(8 \mathrm{~nm})=0.33$. The spatial overlap was set to $\Delta x_{d}=2.5 \mathrm{~mm}$, which corresponds to $42 \%$ of the beam diameter for the third harmonic and $33 \%$ for the fundamental, respectively. The main diagram in Fig. 12 shows the correlation of the visibilities between the fundamental and the third harmonic pulses. Two different delays between the partial beams of $\tau=-1.5 \mathrm{fs}$ (red dots) and $\tau=+2.5$ fs (green dots) have been tested. While the visibility of the fundamental varies only from $V=0.6$ to 0.85 , that of the third harmonic shows values between $V=0.075$ and $V=0.375$. Remarkably, data points towards the upper end in both visibilities represent FLASH pulses which show nearly a perfect spatial coherence with only a few modes contributing. At the lower end of the visibilities significantly more modes are present resulting in a suppressed coherence for the third harmonic. It should be noted that the recorded visibilities are susceptible also to fluctuations of the temporal coherence, since these experiments were performed at finite delays. Because the average temporal coherence of the fundamental with $\tau_{c}=6$ fs is significantly longer than that of the harmonic $\left(\tau_{c}=2.4 \mathrm{fs}\right)$, this results in larger visibility fluctuations for the third harmonic. Therefore, these correlations will be the subject of further investigations.

\section{CONCLUSION}

In this paper a detailed study of the coherence properties of free-electron-laser radiation at FLASH lasing at different wavelengths from $\lambda=32 \mathrm{~nm}$ down to $\lambda=8 \mathrm{~nm}$ is presented. A monotonous decrease of coherence time from $\tau_{c}=6$ fs at $\lambda=32 \mathrm{~nm}$ to $\tau_{c}=2.9$ fs at $\lambda=8 \mathrm{~nm}$ fundamental wavelength is measured. The temporal coherence is found to scale with $\lambda^{1 / 2}$ which is in good agreement with FEL theory. A transverse coherence length for $\lambda=24 \mathrm{~nm}$ of $2.3 \mathrm{~mm}(\mathrm{rms})$ at the entrance of the autocorrelator is observed, where the beam size is $2.5 \mathrm{~mm}$ (rms). For the spatial coherence a Gaussian mode decomposition is performed showing that the transverse coherence properties can sufficiently be described by six modes contributing to the radiation field, where the first three modes contribute more than $90 \%$ to the radiated intensity. Furthermore, the correlation of the visibility between the fundamental radiation at $24 \mathrm{~nm}$ and its third harmonic at $8 \mathrm{~nm}$ is investigated for identical shots. While the visibility of the fundamental varies only from $V=0.6$ to 0.85 , that of the third harmonic shows values between $V=0.075$ and $V=0.375$. 


\section{ACKNOWLEDGMENTS}

The authors gratefully acknowledge the support by the FLASH and HASYLAB teams. We would like to thank M. Yurkov for elucidating discussions. Technical contributions by S. Eppenhoff and the mechanical workshop of the University of Münster have been essential. Financial support occurred by the Bundesministerium für Bildung und Forschung via Grant No. 05-KS4PMC/8 within the research program FSP 301 "FLASH".

[1] W. Ackermann et al., Nat. Photon. 1, 336 (2007).

[2] P. Emma et al., Nat. Photon. 4, 641 (2010).

[3] H. Chapman et al., Nature Phys. 2, 839 (2006).

[4] C. M. Günther, B. Pfau, R. Mitzner, B. Siemer, S. Roling, H. Zacharias, O. Kutz, I. Rudolph, D. Schondelmaier, R. Treusch, and S. Eisebitt, Nat. Photon. 5, 99 (2011).

[5] C. Bostedt et al., Phys. Rev. Lett. 100, 133401 (2008).

[6] B. Siemer, T. Hoger, M. Rutkowski, R. Treusch, and H. Zacharias, J. Phys. Condens. Matter 22, 084013 (2010).

[7] R. Bonifacio, C. Pellegrini, and L. M. Narducci, Opt. Commun. 50, 373 (1984).

[8] E. L. Saldin, E. A. Schneidmiller, and M. V. Yurkov, Opt. Commun. 186, 185 (2000).

[9] E. L. Saldin, E. A. Schneidmiller, and M. V. Yurkov, Phys. Rev. ST Accel. Beams 9, 030702 (2006).

[10] E. L. Saldin, E. A. Schneidmiller, and M. V. Yurkov, The Physics of Free Electron Lasers (Springer, Berlin, 2000).

[11] E. Saldin, E. Schneidmiller, and M. Yurkov, Opt. Commun. 281, 1179 (2008).
[12] R. Mitzner et al., Phys. Rev. A 80, 025402 (2009).

[13] U. Frühling et al., Nat. Photon. 3, 523 (2009).

[14] R. Mitzner et al., Opt. Express 16, 19909 (2008).

[15] W. F. Schlotter, F. Sorgenfrei, T. Beeck, M. Beye, S. Gieschen, H. Meyer, M. Nagasono, A. Föhlisch, and W. Wurth, Opt. Lett. 35, 372 (2010).

[16] A. Singer, I. A. Vartanyants, M. Kuhlmann, S. Duesterer, R. Treusch, and J. Feldhaus, Phys. Rev. Lett. 101, 254801 (2008).

[17] E. L. Saldin, E. A. Schneidmiller, and M. V. Yurkov, Nucl. Instrum. Methods Phys. Res., Sect. A 562, 472 (2006).

[18] J. W. Goodman, Statistical Optics (Wiley InterScience, New York, 1985), 1st ed.

[19] I. A. Vartanyants and A. Singer, New J. Phys. 12, 035004 (2010).

[20] L. Mandel and E. Wolf, Optical Coherence and Quantum Optics (Cambridge University Press, Cambridge, 1995).

[21] P. Schmüser, M. Dohlus, and J. Rossbach, Ultraviolet andSoft X-ray Free-Electron Lasers: Introduction to Physical Principles, Experimental Results, Technological Challenges (Springer, Berlin, 2008).

[22] R. Bonifacio, L. De Salvo, P. Pierini, N. Piovella, and C. Pellegrini, Phys. Rev. Lett. 73, 70 (1994).

[23] B. W. J. McNeil and N. R. Thompson, Nat. Photon. 4, 814 (2010).

[24] Z. Huang and K. J. Kim, Phys. Rev. ST Accel. Beams 10, 034801 (2007).

[25] J. Feldhaus, J. Arthur, and J. B. Hastings, J. Phys. B 38, S799 (2005).

[26] E. L. Saldin, E. A. Schneidmiller, and M. V. Yurkov, Nucl. Instrum. Methods Phys. Res., Sect. A 475, 92 (2001). 